\title{
Exploring the pedagogical design features of the flipped classroom in undergraduate nursing education: a systematic review
}

\author{
Punithalingam Youhasan ${ }^{1,2^{*}}$ (D), Yan Chen ${ }^{1}$, Mataroria Lyndon ${ }^{1}$ and Marcus A. Henning ${ }^{1}$
}

\begin{abstract}
Background: In recent years, technological advancement has enabled the use of blended learning approaches, including flipped classrooms. Flipped classrooms promote higher-order knowledge application - a key component of nursing education. This systematic review aims to evaluate the empirical evidence and refereed literature pertaining to the development, application and effectiveness of flipped classrooms in reference to undergraduate nursing education.

Methods: A PRISMA systematic review protocol was implemented to investigate the literature pertaining to the development, implementation and effectiveness of flipped classroom pedagogy in undergraduate nursing education. Seven databases (Scopus, PsycINFO, CINAHL, ERIC, MEDLINE, Cochrane, Web of Science) were utilised to survey the salient literature. Articles were appraised with respect to their level of evidence, the origin of study, study design, the aims/s of the study, and the key outcomes of the study. A qualitative synthesis was then conducted to summarise the study findings.
\end{abstract}

Results: The initial search identified 1263 potentially relevant articles. After comprehensively reviewing the initial catchment using several analytical phases, 27 articles were considered for the final review, most of which were conducted in the USA and South Korea. A range of research designs were applied to measure or discuss the outcomes and design features of the flipped classroom pedagogy when applied to undergraduate nursing education. The review indicated that a common operational flipped classroom model involves three key components, namely pre-classroom activities, in-classroom activities and post-classroom activities, guided by two instructional system design principles. The review predominantly identified positive learning outcomes among undergraduate nursing students, after experiencing the flipped classroom, in terms of skills, knowledge and attitudes. However, a few studies reported contrasting findings, possibly due to the incompatibility of the flipped classroom pedagogy with the traditional learning culture.

\footnotetext{
*Correspondence: youhasanp@esn.ac.lk; p.youhasan@auckland.ac.nz 'Centre for Medical and Health Sciences Education, Faculty of Medical and Health Sciences, The University of Auckland, Auckland, New Zealand ${ }^{2}$ Department of Medical Education \& Research, Faculty of Health-Care Sciences, Eastern University, Sri Lanka, Batticaloa, Sri Lanka
} 
(Continued from previous page)

Conclusions: Current evidence in this systematic review suggests that incorporating the flipped classroom pedagogy could yield positive educational outcomes in undergraduate nursing education. There are promising pedagogical models available for adapting or developing the flipped classroom pedagogy in undergraduate nursing education.

Keywords: Flipped classroom, Blended Learning, Inverted classroom, Nursing education, Systematic review, Design principles

\section{Background}

Globally, nursing educational institutions are taking steps forward in redesigning their curricula to align them with modern pedagogy to enhance student-centred learning [1]. This curricular reform is concerned with fulfilling the educational needs of the new technological era, which generates exposure to a variety of information, advanced communication technology, and diverse learning methods [2]. As a result, blended learning has become part of this curricular reform. Blended learning is a broad pedagogical approach, which encompasses a combination of face-to-face and online teaching to promote student-centred learning [3]. One of the blended learning innovations is the notion of the flipped classroom [4], referred to as "a hybrid approach to learning, using technology to move the classroom lecture to homework status and using face-to-face classroom time for interactive learning" [5]. The rule of thumb of the flipped classroom is redesigning the face-to-face classroom as an interactive learning environment where higher-ordered learning takes place, while providing traditional pedagogical experiences (of transferring basic information) through pre-class learning activities [4, 68]. As such, pre-class learning materials can be provided to closely represent learning in the traditional face-toface classroom but being delivered electronically or via online media [9]. Therefore, pre-learning materials should be accompanied with teachers' explanation rather than relying on the sole use of pre-class reading materials [6].

Contemporary nursing care is advancing dramatically due to the need for nursing students to manage problems associated with multiple and complex clinical comorbidities [10]. It was reported in the literature that nursing students experience difficulties in applying learnt knowledge in clinical practice [11]. This necessitated the need for nursing curricular implementers to adopt pedagogies like the flipped classroom to ensure that theoretical concepts were explicitly linked to patient care. As such, the flipped classroom is considered as a new educational paradigm for implementing health professions' education curricula $[6,12]$. While there are increasing applications of the flipped classroom, there is a dearth of evidence evaluating its impact on student learning and curriculum design in undergraduate nursing education [13-15]. The empirical evidence to date has predominantly examined the effectiveness of the flipped classroom through students' satisfaction and academic performance [13]; however, there is limited evidence to explain the pedagogical design principles of the flipped classroom, which are indispensable to achieving meaningful educational effectiveness [16]. Thus, the following systematic review aims to describe and evaluate research conducted in the area of designing, developing and implementing the flipped classroom, and appraise the educational impact of the flipped classroom approach when applied to undergraduate nursing education.

\section{Methods}

This systematic review was performed in accordance with the PRISMA (Preferred Reporting Items for Systematic Reviews and Meta-Analyses) protocols (Additional File) [17]. PRISMA is an evidence-based system used to guide reporting in systematic reviews and metaanalyses [18]. The protocol was registered with the PROSPERO (International prospective register of systematic reviews) (CRD42020194474, 16th October 2020).

\section{Systematic literature search}

A literature search was conducted utilising seven databases (PsycINFO, CINAHL, ERIC, MEDLINE, Web of Science, Cochrane Library and Scopus) in November 2019. The key concept of the literature search was the term flipped classroom. This term was combined with a range of supplementary key words relevant to nursing education using a PICOS (Population, Intervention, Comparison, Outcomes and Study) framework [19]. The derived terms were: Population - Undergraduate Nursing Students; Intervention - Flipped Classroom; Comparison - Traditional Classroom; Outcomes - Educational achievements and pedagogical designs; and Study - any original research studies. A search algorithm was created by using keywords with Boolean operators to conduct a literature search in the databases. A sample search strategy in MEDLINE is illustrated in Table 1. 
Table 1 The MEDLINE search strategy and term used

\begin{tabular}{|c|c|c|}
\hline Search & Algorithms & Article $(\mathrm{n})$ \\
\hline 1 & $($ flip* adj2 (class* or learning or teaching or pedagog*)) & 483 \\
\hline 2 & (invert* adj2 (class* or learning or teaching or pedagog*)) & 205 \\
\hline 3 & $\begin{array}{l}\text { (nursing edu* or nurs* edu* or nurs* or teach* nurs* or health profession* } \\
\text { education* or health person* or health person* education* or health occupation* } \\
\text { or health occupation*education*) }\end{array}$ & 517,704 \\
\hline 4 & (undergrad* or baccalaureate or bachelor* or student*) & 321,077 \\
\hline 5 & (Search-1) or (Search-2) & 674 \\
\hline 6 & (Search-3) or (Search-4) & 594,770 \\
\hline 7 & (Search-5) and (Search-6) & 413 \\
\hline 8 & (Search-7) limited to (year = "2012 -Current" and English) & 374 \\
\hline
\end{tabular}

\section{Study selection}

Titles of the manuscripts which were identified in the database search were transferred to a bibliography management programme (Endnote X9, Thomson Reuters, New York) to create a search library and remove duplicates. The resulting studies were independently and systematically reviewed by an author (PY) in accordance with the inclusion criteria (Table 2), first by title and then by abstract. Then, full texts of the selected studies were again reviewed by the author (PY) and he made a $\log$ of all reviewed studies with reasons for inclusion or exclusion. The log was cross-checked by the other three authors (YC, ML \& MAH). Following this, all four authors met at various times to discuss and review all chosen articles. Any disagreements were resolved through discussions within the whole research group until a consensus was reached. Moreover, citations from the selected studies were scrutinised to confirm that all relevant studies were identified.

\section{Data synthesis}

The data synthesis was performed using an electronic data extraction table (in Microsoft Excel). The following details were extracted from each reviewed study: name of authors, country, publication year, participants, research design, research procedure, research instrument, analysis of data, key findings and conclusion. The initial data extraction was completed by PY. The extracted data were independently reviewed for accuracy by the other three authors (YC, ML, \& MAH), This group confirmed the inter-rater reliability and resolved any outstanding issues, such as data entry errors. Furthermore, if the details from a selected study was inadequate or ambiguous, additional information was obtained from the corresponding author/s of the relevant study. Lastly, an inductive thematic method was used to analyse the extracted (qualitative) data [20]. This process incorporated a series of inductive stages. First, the extracted data were line-by-line coded by the first author (PY). Then, the codes were crossed checked (by all authors) and clustered under descriptive themes. Finally, the descriptive themes were further condensed into analytical themes to provide an in-depth description regarding the aims of the review. With the exception of the first step, all other steps were conducted in a meeting with the presence of all four authors for establishing inter-rater reliability.

\section{Quality assessment of the selected studies}

An evidence hierarchy classification model (Table 3) was used to assess the quality of the studies [21-23]. Each publication included in the data synthesis was then allocated to an evidence hierarchy classification (I to IV). Subsequently, the publication was assigned to the operational ranks as devised by Jensen et al. (2004) [22]. To maintain the integrity of the quality assessment process, evidence appraisals were independently rated by two authors (PY \& MAH). The ratings were presented and

Table 2 Inclusion and exclusion criteria for selecting articles

\begin{tabular}{|c|c|}
\hline Inclusion Criteria & Exclusion Criteria \\
\hline $\begin{array}{l}\text {-Description of the Flipped classroom (pedagogy/learning/teaching) in } \\
\text { nursing education. } \\
\text {-Study using any form of pedagogical model/framework. } \\
\text {-Study focusing on measuring the effectiveness of flipped classroom } \\
\text { pedagogy. } \\
\text {-Study conducted in undergraduate education. } \\
\text {-The publication period from } 2012 \text { to } 2019 \text { (The flipped classroom was } \\
\text { introduced into Health Profession Education in } 2012 \text { [6]). } \\
\text {-Type of publication: Original research, systematic review, or meta- } \\
\text { analysis. }\end{array}$ & $\begin{array}{l}\text { - Full text of the article is not published in English. } \\
\text {-Study conducted in the context of post-graduate and vocational training. } \\
\text {-Study results duplicated in a separate earlier publication. } \\
\text {-Type of publication: book, chapters, thesis, commentaries, conference } \\
\text { abstracts, protocols, study outlines and government publication. }\end{array}$ \\
\hline
\end{tabular}


Table 3 Categories of evidence and its definitions

\begin{tabular}{lll}
\hline Categories & Definitions & Operational ranks \\
\hline la & Evidence from meta-analysis of randomized controlled trials & Rank A \\
Ib & Evidence from at least one randomized controlled trial & \\
Ila & Evidence from at least one controlled study without randomization & Evidence from at least one other type of Quasi-experimental study \\
IIb & Evidence from non-experimental descriptive studies, such as comparative studies, \\
III & correlation studies, case-control studies and qualitative studies. & Rank B-D \\
IV & Evidence from expert committee reports or opinions and / or clinical experience of respected authorities & \\
\hline
\end{tabular}

discussed amongst all four authors in a meeting to finalize the allocation of category of evidence.

\section{Results}

\section{Study selection}

The initial search yielded a total of 1263 hits from the seven databases $($ PsycINFO $=53, \mathrm{CINAHL}=145, \mathrm{ERIC}=$ 361, MEDLINE $=374$, Web of Sci. $=196$, Cochrane $=10$, Scopus $=124)$. One hundred and sixty duplicates were identified, and 1103 studies were considered for title and abstract screening. In this title and abstract screening, 629 studies were excluded as they were deemed out of scope. The subsequent quota of studies $(n=474)$ was included for assessing the full texts. A list of 104 studies was identified as potentially relevant to the systematic literature review by three authors. Further, this was reduced to a final list of 27 refereed sources after appraisal of the full texts (Fig. 1). The key study features of the 27 articles in the evidence synthesis are presented in Table 4.

\section{Study characteristics Study participants}

Participants of the study were defined as undergraduate students who enrolled in the nursing programme. Eleven studies reported the details of students' academic year of study. Accordingly, the academic year of participating nursing students range from 1 st to 4 th year.

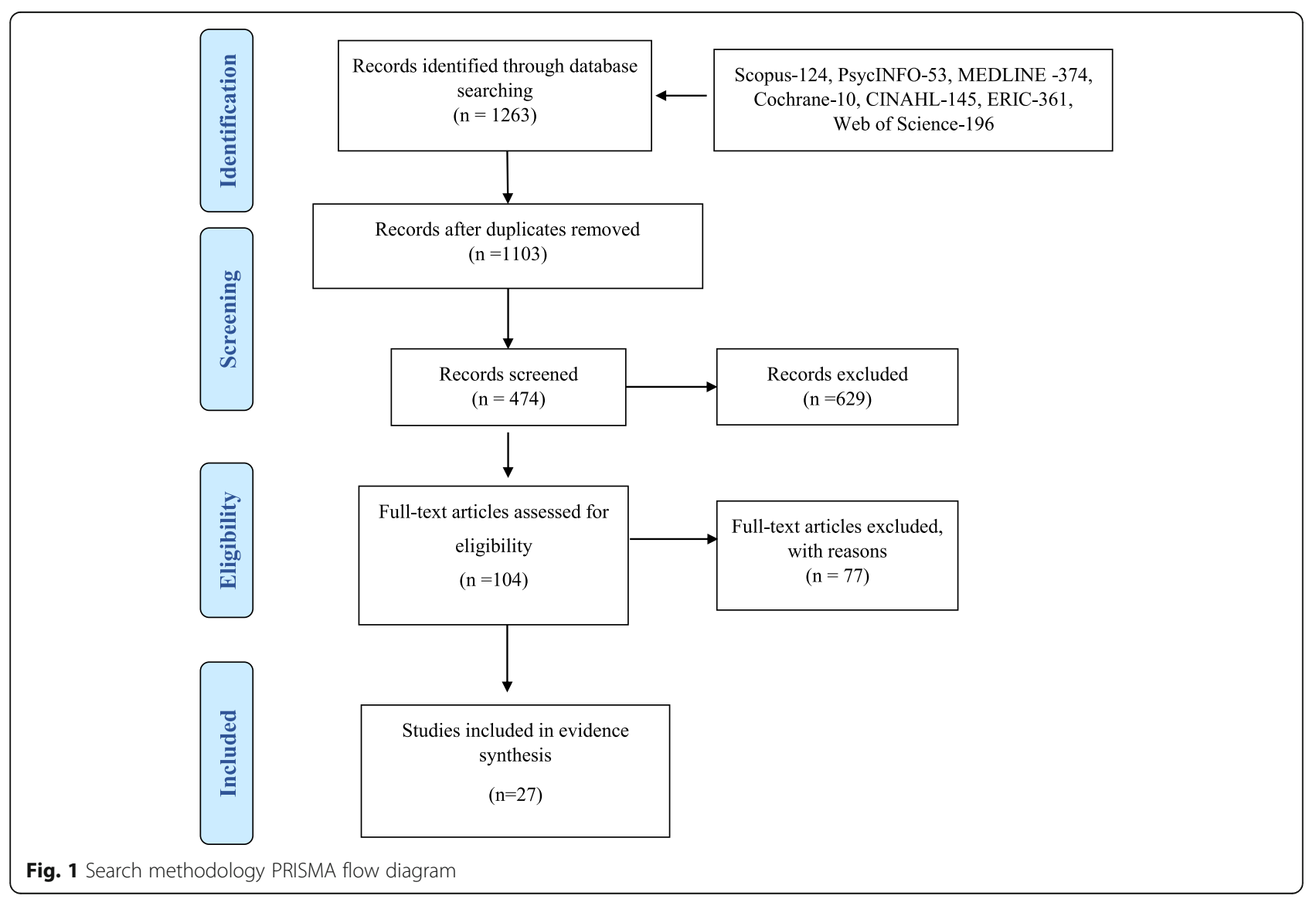




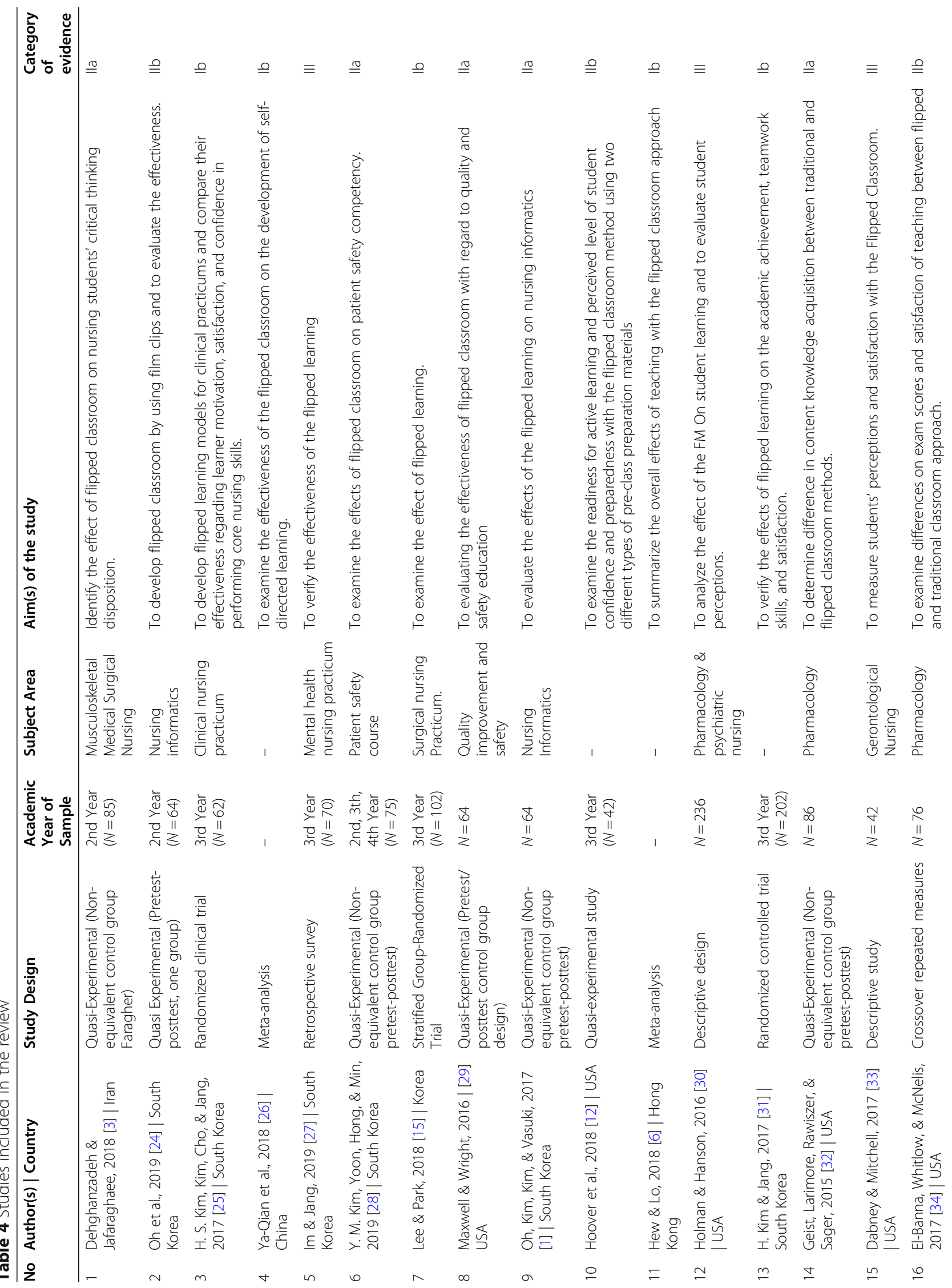




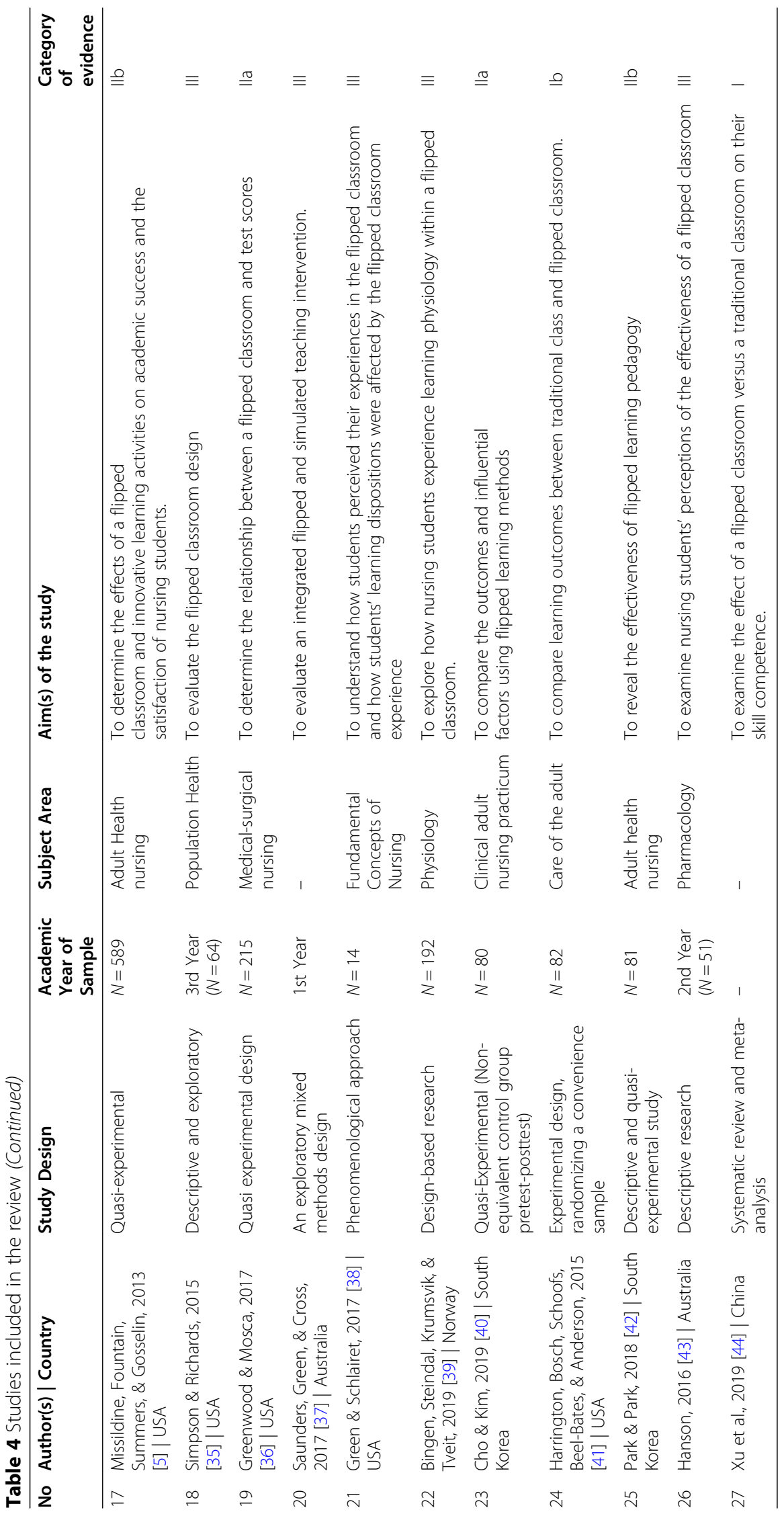


Nevertheless, most of the studies $(n=6)$ were conducted among third-year nursing students (Table 4).

Demographic information of the participants, including age and gender, was included in 12 studies. Two further studies only included the age of participants, while an additional study reported only gender. However, 12 studies did not report demographic variables. The majority of the reported participants were females, and the mean age range was from 19 to 31.5 years. Sample size was reported in the 23 studies which, ranged from 14 to 589.

\section{Study setting}

The selected studies were primarily conducted in the context of tertiary level nursing education. Most of the studies were conducted in the USA $(n=11,40.7 \%)$, followed by South Korea $(n=9,30.3 \%)$. Two articles each were found to be published from Mainland China $(n=2)$ and Australia $(n=2)$. One article was published from Norway, Iran and Hong Kong. Moreover, the flipped classroom experiences were reported in reference to a vast range of nursing subjects or courses (Table 4).

\section{Methodical quality of studies}

According to the evidence hierarchy classification, the majority of the articles $(n=19)$ were IIb $(n=5)$ or above $(n=14)$. The review also included one Ia category evidence [44]. Interestingly, according to operational ranks, all the articles which were included for the review were clustered into rank A (Table 4).

\section{Evidence synthesis on the flipped classroom in nursing education}

\section{Qualitative thematic synthesis of findings}

The thematic synthesis revealed 37 codes. The identified codes were clustered into four descriptive themes; namely, knowledge and skills; attitudes and perceptions; flipped classroom (FC) design; and teaching and learning (TL) strategies. The descriptive themes were further specified to two analytical themes for providing profound insights and excelling the context of the present review. The analytical themes revealed were i) the pedagogical structure of the flipped classroom and ii) influence of flipped classroom on nursing students' learning (Fig. 2).

\section{Analytical theme 1: pedagogical structure of the flipped classroom}

The systematic review revealed that the flipped classroom is a new educational paradigm in undergraduate nursing education $[6,12]$. A common operational flipped classroom model (Fig. 4) was reported in the selected literature which consisted of three components, namely pre-classroom activities, in-classroom activities and post-classroom activities $[1,15,25,27,28,31,33,35$,
38-40, 42, 43]. Pre-class activities require the provision of learning material by educators to students [25]. The learning material is mainly transferred through an online learning management system to students [1, 24, 25], at least one week before the face-to-face classroom session $[3,27,28]$. The pre-learning materials can be delivered in various forms, such as video lecture, narrated PowerPoint, animation (illness scenario) and video demonstrations of the nursing procedure $[1,25,36,38]$ with further readings $[33,35,38,42]$. The duration of the video learning material ranged from 10 to $20 \mathrm{~min}$ [15, 27, 31]. Different software were used to create prelearning materials, such as "Articulate Storyline" [3], "Explain Everything" [27], "Camtasia Studio" [12]. At the end of pre-class activities, an assessment was conducted mainly as quizzes [28, 31, 35, 39]. Furthermore, students were able to interact with teachers and peers through online dashboards [15, 24, 25, 28, 39].

The in-classroom learning environment was designed as an interactive space for applying, analysing and evaluating the pre-learning material [1]. For in-classroom activities, students were divided into small groups $[3,31$, $36,38-40]$ and the reported group size ranged from two to six $[3,25,38,42]$. Some studies used quizzes as a diagnostic test at the beginning of the in-class activities $[3,12,43]$, followed by several student-centred learning activities $[3,5,27,28,31,35,36,38]$. Other studies reported that teachers conducted a micro-lecture for summarizing and clarifying complex phenomena [12, 27, 40, 42, 43].

Post-class activities continued with a follow-up discussion of the newly learnt concepts or issues which had not been solved in the previous in-class session [1, 25]. The follow-up discussions were mainly conducted online [1]. Post-class tests can be conducted to assess students' learning [3, 42]. Finally, at the end of the flipped classroom experience, students completed a survey to evaluate the effectiveness of the flipped classroom [12, 31].

In terms of developing a flipped classroom, two studies were identified that investigated instructional system designs. Lee and Park (2018) outlined nine design principles for developing a flipped classroom that could be used in a surgical nursing practicum [15]. These are illustrated in the Fig. 3.

Oh et al. (2019) used a framework termed the ADDIE model for developing a flipped classroom to teach a nursing informatics course [24]. This model has been used in terms of its five straightforward steps: Analyze, Design, Develop, Implement, and Evaluate (Fig. 4). First, the Analyze step involves the assessment of feasibilities for adopting flipped classroom in terms of current practice, equipped environment, stakeholder's readiness and nature of the curriculum. The Design phase deals with framing the 


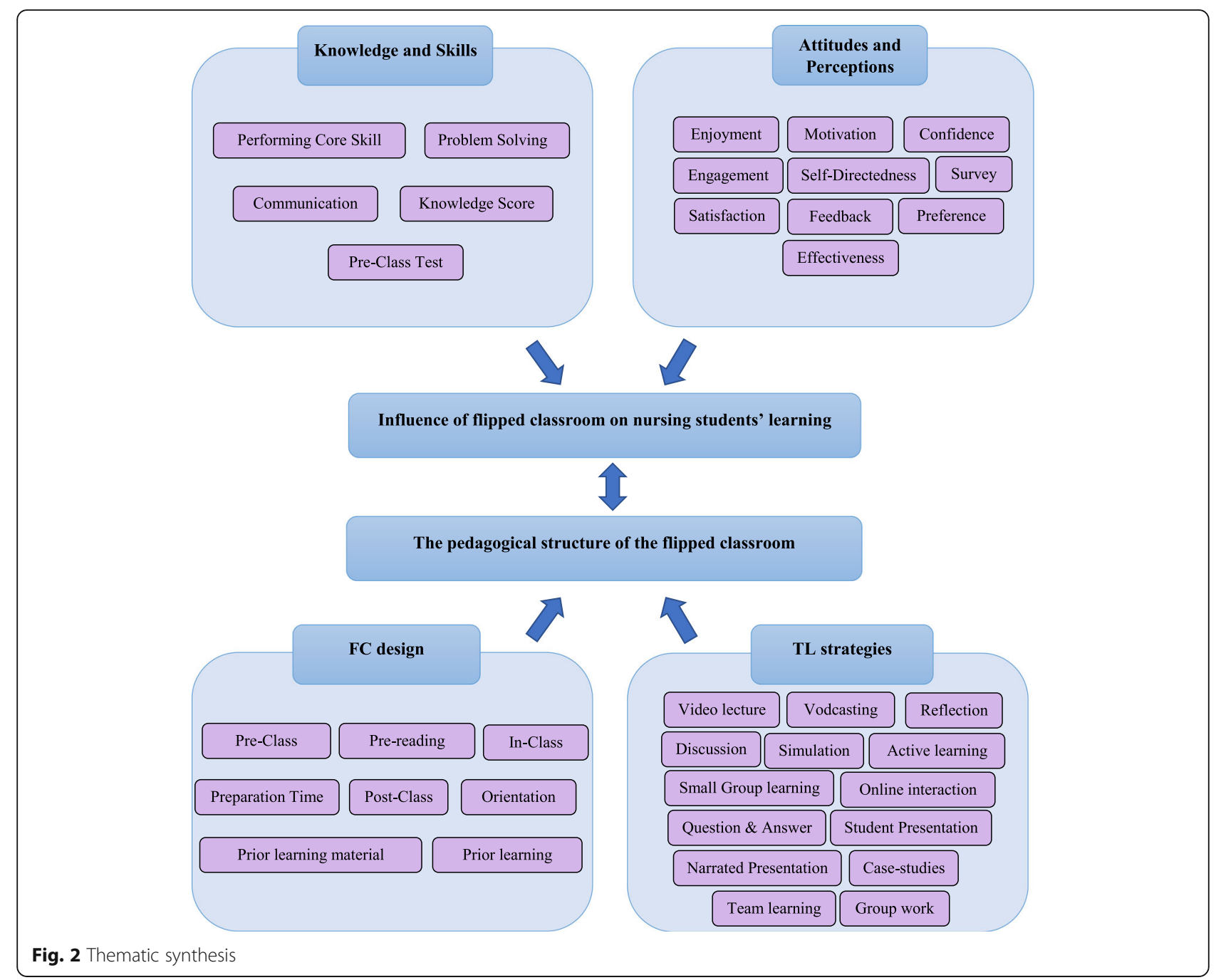

instructional strategies, such as identifying courses for implementation, defining the operational procedures, lesson planning, choosing assessment instruments, designing the user interface and choosing the audiovisual designs. The Development phase starts with the production of teaching-learning material for the flipped classroom. In addition, an instrument is developed for measuring the effectiveness of the flipped classroom on students' learning. The Implementation phase requires participants to receive the flipped classroom. The last step of the ADDIE method is Evaluation. The main aims of this phase are to gather feedback from participants and assess the educational improvement of the learners to quantify the effectiveness of the intervention and identify the way forwards for future improvement [24].

Influence of flipped classroom on nursing students' learning Several studies addressed the effect of flipped classroom learning on the students' knowledge and skill.
Oh et al. (2019) revealed that integrating flipped classroom led to a significant improvement in nursing students' test scores [24]. The same impact was observed in another seven studies $[5,28,29,31,32,36$, 42]. In contrast, four studies concluded that flipped classroom learning does not influence students' assessment performance [30, 34, 35, 41].

Six studies reported the influence of flipped classroom on nursing students' skill development. Kim and Jang (2017) revealed that students' clinical performance evaluation score increased after 10 weeks of a flipped classroom intervention [31]. Similarly, nursing students' core competencies in clinical practicum increased after the flipped classroom experience in a clinical setting [27]. The above two findings were endorsed by a metaanalysis of 22 randomised controlled trials, reporting that flipped classroom improved nursing students' skill competence [44]. The effect was further confirmed by two studies indicating that the flipped classroom approach increased nursing students' confidence in 


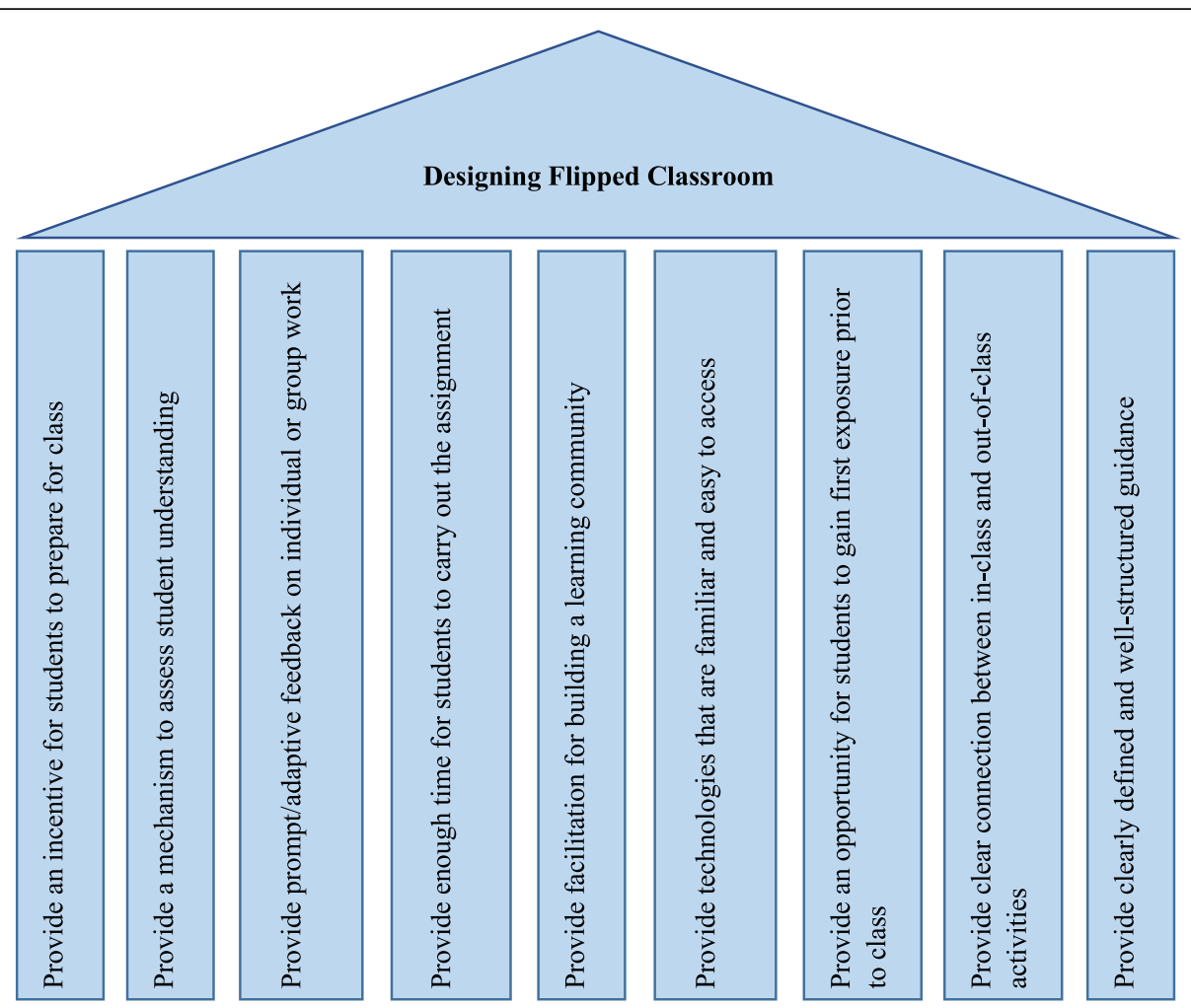

Fig. 3 Nine design principles for developing flipped classroom [15]

performing core skills [25, 40]. Kim et al. (2019) reported that nursing students' patient safety competency was significantly increased after the flipped classroom experience [28]. In terms of problem-solving skill, Lee and Park (2018) concluded that nursing students who received the flipped classroom showed significant improvements in problem-solving skills [15]. A study investigated communication skills, reported that the outcome of therapeutic communication was significantly increased among the nursing students, after attending the flipped classroom on the mental health nursing practicum [27].

There were several noticeable attitudinal changes among nursing students due to the flipped classroom, namely satisfaction, motivation, engagement, confidence, selfdirectedness, enjoyment, and critical thinking. In terms of satisfaction, four studies reported that flipped classroom learning increased nursing students' satisfaction $[1,25,31$, 37]. In contrast, one study noted that nursing students expressed more satisfaction with the traditional lecturebased learning model than flipped classroom learning [30]. In addition, a study reported that nursing students' satisfaction plummeted at the initial period with the introduction of the flipped classroom [36]. Moreover, nursing students with kinesthetic learning styles were satisfied with the flipped classroom while learners classified as having a preference for visual and auditory stimuli preferred traditional teaching methods [38].
In relation to self-directed learning, four studies reported that flipped classroom pedagogy enhanced selfdirected learning among nursing students $[1,26,30,40]$. Self-goal setting ability was significantly increased among nursing students who attended a flipped classroom [15]. Regarding nursing students' motivation, two studies indicated that the flipped classroom enhanced learning motivation $[1,26]$. In reference to nursing students' learning engagement, the flipped classroom was recognised as an active learning method which enhanced learning engagement [35]. In addition, flipped classroom approaches improved nursing students' cooperative spirit and teamwork, which increased their interest to engage in learning [44].

Four studies found the flipped classroom an enjoyable way of learning in nursing education [1, 33, 35, 44]. It is interesting to note that nursing students enjoyed viewing the video lecture more than the live lecture [33]. In terms of critical thinking, flipped classrooms increased nursing students' critical thinking [3, 42]. Moreover, the flipped classroom enabled nursing students' ability to think deeply and analyse the problem [43, 44].

\section{Discussion}

This systematic review explored and evaluated the flipped classroom in the context of undergraduate nursing education. In particular, the systematic review 


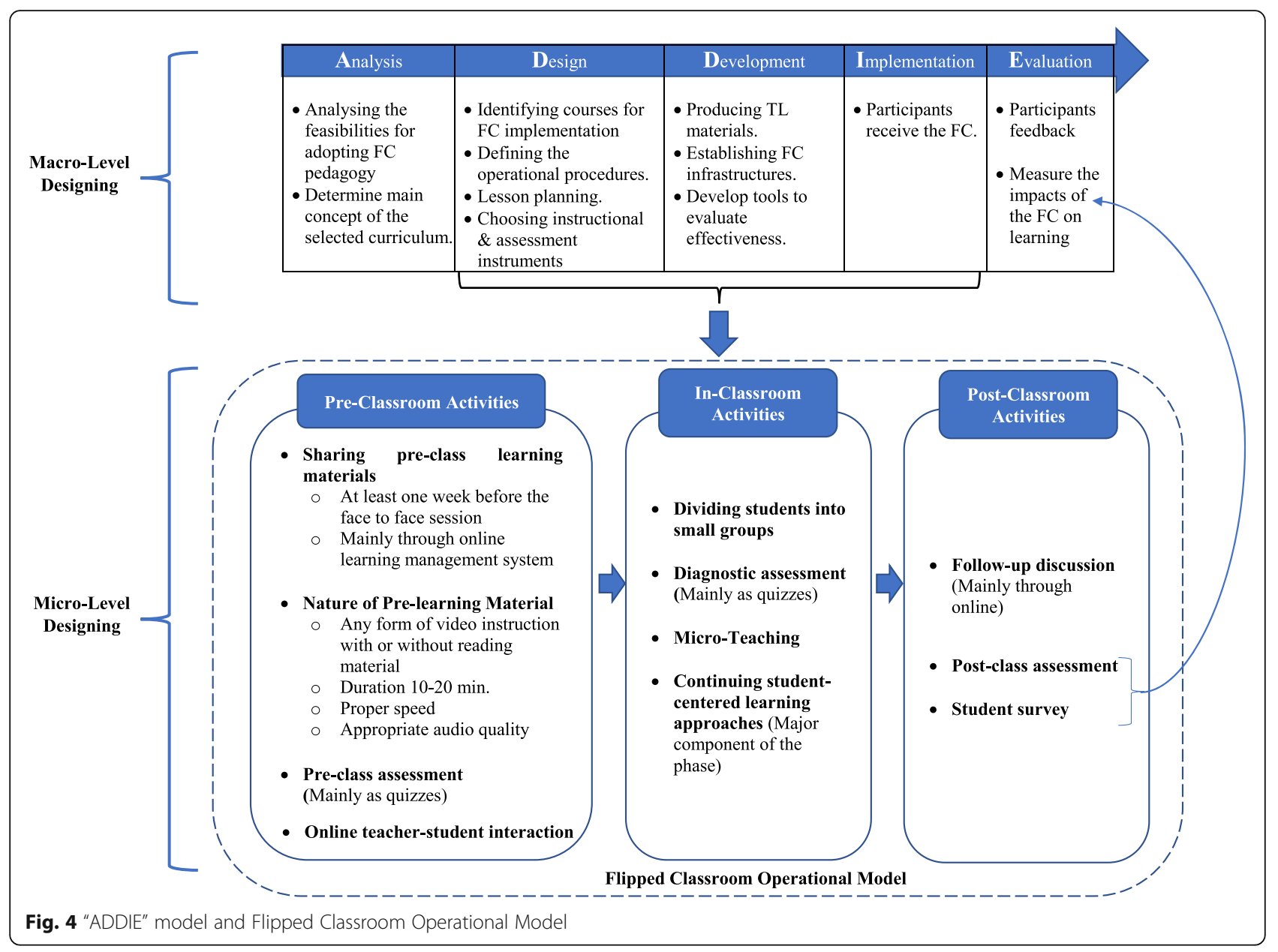

addressed two main aspects - one focusing on the design and development of flipped classroom pedagogy in undergraduate nursing education and the other evaluating the impact of the flipped classroom on undergraduate nursing students' learning.

The systematic review identified 27 studies that investigated the flipped classroom experience among undergraduate nursing students. According to the evidence hierarchy classification model $[21,22]$, most of the selected studies reached the evidence category of IIb or above and all of them achieved operational rank "A", indicating a catchment of high quality papers. A variety of methodologies, including educational measures were used to determine the impact of the flipped classroom on undergraduate nursing students' learning. As the studies varied significantly, it is not easy to perform a direct comparison between studies due to the degree of heterogeneity. Nevertheless, the results of the selected studies revealed that a common operational pedagogical structure (Fig. 4) was generally utilised regardless of instructional system designing principles (except for two studies) and the flipped classroom resulted in positive learning outcomes among undergraduate nursing students.
Taking the flipped classroom design into consideration, studies investigated the flipped classroom design in reference to both micro and macro levels [24, 45]. The micro level concerns developing flipped classroom pedagogy for a session or topics $[45,46]$. In contrast, the macro level involves instructional system design at the curriculum or course level $[45,47]$. Most of the selected studies included in this review examined the flipped classroom at the micro level. Interestingly, the three-step flipped classroom operational model (Fig. 4) describes the flipped classroom design at the micro-level. It was noted that the common operational model was utilised in different forms. For example, Oh et al. (2019) used the basic operational model in eight steps which is called the "C-REVERSE" design with the use of film clips [24] and "flipped-mastery classroom model" was used in the South Korean clinical nursing practicum curriculum [25]. However, the existing findings emphasize that the benefits of the flipped classroom did not eventuate based on sticking purely to the common operational model, but rather caused by the logical connections between the different steps $[45,48,49]$. 
Some studies included the pre-classroom activities and post-classroom activities under a common cluster of online-learning phase and in-classroom activities labelled as face-to-face learning phase [1, 39, 43, 45, 50]. Three important concerns were reported for developing the online learning phase [45, 46, 51] through evaluating the: (i) physical feature of the video or online lectures which includes duration, pacing and quality of audio; (ii) content feature of the video or online lecture such as appropriate provision of the online portion, clarity and interactivity; and (iii) logistic feature of the video or online lecture namely formative assessment, timetabling and follow-up activities. Designing the face-to-face learning phase is crucial because it is the core part of the flipped classroom [45]. The current review suggests four cardinal activities of the in-class activities, namely dividing students into small groups, conducting a diagnostic assessment, micro-teaching, and continuing integrative student-centred instructions. Furthermore, it has been suggested that the face-to-face learning phase should include: (i) introductory tasks such as minilecture and authenticating quizzes; (ii) interactive learning activities which are aligned with the intended learning outcomes; and (iii) well established ground rules and learning culture $[45,46,52,53]$.

In terms of macro-level design, the review identified that the ADDIE model created a framework for designing the flipped classroom for undergraduate nursing students [24]. The ADDIE model has been recognized as effective, systematic and efficient in designing the flipped classroom in nursing education [54, 55]. Moreover, the ADDIE model has achieved acceptance in diverse fields $[56,57]$. The ADDIE model proposes five straightforward steps when developing the pedagogical strategies used ensure curriculum planners and implementers reach the 'appropriate destination' [56]. In addition, the review traced the design principles of the flipped classroom. Lee and Park (2018) adopted nine design principles (Fig. 3) for developing flipped classrooms in reference to the surgical nursing practicum [15]. Kim et al., (2014) proposed the flipped classroom design principles for enforcing student-centred learning through four key variables, namely cognitive presence, social presence, teaching presence, and learner presence [58].

In reviewing the impact of the flipped classroom on undergraduate nursing students' learning, positive outcomes were reported in many studies included in this review. More specifically, nursing students' knowledge, skills and attitudes were improved by the flipped classroom learning, in terms of assessment performance, performing core skills, problem-solving, communication, critical thinking, self-directedness, motivation, engagement, confidence, satisfaction, and joyful learning. Besides, the notions of positive outcomes were reported among students from other discipline such as dentistry, medicine, pharmacy [59-63]. It was reported from the literature that two main explanations contributed to the positive learning outcomes. Firstly, unimpeded access to the pre-classroom learning materials enabled nursing students to learn in their preferred place, pace and time. Specifically, the pre-recorded video lecture was used as the main pre-classroom learning material. The nursing students who watched the video lectures developed a better understanding of learning concepts [6]. Secondly, in-classroom activities were designed as an interactive and student-centred environment which provided greater opportunity to apply the learned concepts into practice [1]. On the other hand, some studies still favoured the traditional lecture-based learning [30, 41]. This may be due to the preference for behaviouristic learning in higher education. Overall, the findings so far seem to suggest that we still have mixed results on whether flipped classroom increases test scores; however, there seems to be strong evidence to suggest that flipped classroom can increase student motivation, satisfactory, and critical thinking.

These findings present two important implications for developing and implementing the flipped classroom in undergraduate nursing education. Firstly, contextual compatibility is more important for the success and sustainability of a pedagogical model. Thus, it is essential to follow an instructional system design at the macro-level to develop flipped pedagogy rather than using its' operational structures alone at the micro-level. The review identified the ADDIE model and the three-step operational model (Fig. 4) for fostering flipped classroom at the macro and micro level, respectively. Secondly, it was noted that the flipped classroom resulted in positive learning outcomes among nursing students. This outcome may be optimised by balancing the workload of pre-, in-, and post-class activities at the micro level, rather than providing more emphasis on one phase. Furthermore, a study reported that the flipped classroom was not welcome by stakeholders during the introduction phase [36]. Consequently, the solidity of the flipped classroom intervention relies on the constant and stable plan of implementation.

This review could have limitations derived from the heterogeneity of study designs. Apart from metaanalysis, randomised controlled studies, and quasiexperimental studies, we also included several nonexperimental descriptive studies to cover the range of available evidence. This heterogenous sample of studies does not permit further probing of the evidence, such a meta-analysis of the study outcomes; however, our sample represents the commonly-used and ethical research methods in educational research and provides a starting point for generating higher levels of evidence. Moreover, 
the reported findings are mainly from the United States of America and South Korea, which are likely wellresourced settings. Consequently, there may be cultural and regional bias in these studies like ethnocentrism, available resources and educational system. Thus, future research could be conducted in other settings, including low and middle-income countries, to strengthen the evidence base.

\section{Conclusions}

The evidence cited in this systematic review suggests that incorporating the flipped classroom pedagogy probably yields promising positive educational outcomes in undergraduate nursing education. The majority of the studies utilized a common operational flipped classroom structure as pre-classroom, in-classroom and postclassroom. Furthermore, there are promising instructional system design models available for adapting or developing a flipped classroom. Practical implications of the review are considering contextual compatibility and providing equal importance to all three phases of flipped classroom for augmenting the educational outcomes. In addition, the feasibilities of developing the flipped classroom in a limited-resourced setting are still inconclusive. Therefore, future research should consider developing and implementing flipped classrooms for the limitedresourced undergraduate nursing educational environment by using a compatible instructional system designing model.

\section{Abbreviations}

PRISMA: Preferred Reporting Items for Systematic Reviews and Meta-Analysis; FC: Flipped Classroom; TL: Teaching and Learning; ADDIE: Analyse, Design, Develop, Implement, and Evaluate

\section{Acknowledgements}

We would like to acknowledge Anne Wilson for her contribution in developing the search algorithm.

\section{Authors' contributions}

All authors contributed to the design of the study and created the search strategy. PY conducted the literature search, screened the titles and abstracts, extracted, analysed, and interpreted the data, drafted the initial manuscript. YC, ML, and MAH critically evaluated the search strategy, contributed to the selection of articles, and made significant contributions to the writing of the manuscript. All authors reviewed and approved the final version of the manuscript and agreed to be accountable for all aspects of the work. The authors read and approved the final manuscript.

\section{Funding}

No grant was obtained for this systematic literature review.

Availability of data and materials

Not applicable.

\section{Declarations}

Ethics approval and consent to participate

Not applicable.

\section{Consent for publication}

Not applicable.

\section{Competing interests}

The authors declare that they have no competing interests.

Received: 8 October 2020 Accepted: 24 February 2021

Published online: 22 March 2021

\section{References}

1. Oh J, Kim SJ, Kim S, Vasuki R. Evaluation of the effects of flipped Learning of a nursing informatics course. J Nurs Educ. 2017;56(8):477-83.

2. Benner P. Educating nurses: a call for radical transformation-how far have we come? J Nurs Educ. 2012;51(4):183-4.

3. Dehghanzadeh $\mathrm{S}$, Jafaraghaee F. Comparing the effects of traditional lecture and flipped classroom on nursing students' critical thinking disposition: a quasi-experimental study. Nurse Educ Today. 2018;71:151-6.

4. Lee Y, Martin KI. The flipped classroom in ESL teacher education: an example from CALL. Educ Inf Technol. 2020;25(4):2605-33.

5. Missildine K, Fountain R, Summers L, Gosselin K. Flipping the classroom to improve student performance and satisfaction. J Nurs Educ. 2013;52(10): 597-9.

6. Hew KF, Lo CK. Flipped classroom improves student learning in health professions education: a meta-analysis. BMC Med Educ. 2018;18(1):38.

7. Persky AM, McLaughlin JE. The flipped classroom - from theory to practice in health professional education. Am J Pharm Educ. 2017;81(6). https:// www.ajpe.org/content/ajpe/81/6/118.full.pdf.

8. FLN. Definition of Flipped Learning Flipped Learning Network 2014 [09.03. 2020]. Available from: https://flippedlearning.org/definition-of-flipped-lea rning/

9. Love B, Hodge A, Corritore C, Ernst DC. Inquiry-based Learning and the flipped classroom model. PRIMUS. 2015;25(8):745-62.

10. Njie-Carr VPS, Ludeman E, Lee MC, Dordunoo D, Trocky NM, Jenkins LS. An integrative review of flipped classroom teaching models in nursing education. J Prof Nurs. 2017;33(2):133-44.

11. Hashemiparast $M$, Negarandeh $R$, Theofanidis D. Exploring the barriers of utilizing theoretical knowledge in clinical settings: a qualitative study. Int J Nurs Sci. 2019;6(4):399-405.

12. Hoover CA, Dinndorf-Hogenson GA, Peterson JL, Tollefson BR, Berndt JL, Laudenbach N. Flipped classroom: do students perceive readiness for advanced discussion? J Nurs Educ. 2018;57(3):163-5.

13. Bernard JS. The Flipped Classroom: Fertile Ground for Nursing Education Research. Int J Nurs Educ Scholar. 2015;12(1):99.

14. Betihavas V, Bridgman $H$, Kornhaber R, Cross M. The evidence for 'flipping out': a systematic review of the flipped classroom in nursing education. Nurse Educ Today. 2016;38:15-21.

15. Lee MK, Park BK. Effects of flipped Learning using online materials in a surgical nursing practicum: a pilot stratified group-randomized trial. Healthc Inform Res. 2018;24(1):69-78.

16. Morrison GR, Ross SM, Kalman HK. Designing effective instruction, 7th edition. Wiley Global Education; 2012.

17. Moher D, Liberati A, Tetzlaff J, Altman DG, The PG. Preferred reporting items for systematic reviews and meta-analyses: The PRISMA statement. PLoS Med. 2009;6(7):e1000097.

18. Moher D, Shamseer L, Clarke M, Ghersi D, Liberati A, Petticrew M, et al. Preferred reporting items for systematic review and meta-analysis protocols (PRISMA-P) 2015 statement. Syst Rev. 2015;4(1):1.

19. Amir-Behghadami M, Janati A. Population, intervention, comparison, outcomes and study (PICOS) design as a framework to formulate eligibility criteria in systematic reviews. Emerg Med J. 2020;37(6):387.

20. Thomas J, Harden A. Methods for the thematic synthesis of qualitative research in systematic reviews. BMC Med Res Methodol. 2008;8(1):45.

21. Eccles M, Freemantle N, Mason J. Using systematic reviews in clinical guideline development. In: Systematic Reviews in Health Care.2001. p. 400-9.

22. Jensen LS, Merry AF, Webster CS, Weller J, Larsson L. Evidence-based strategies for preventing drug administration errors during anaesthesia. Anaesthesia. 2004;59(5):493-504.

23. Webster CS, Luo AY, Krägeloh C, Moir F, Henning M. A systematic review of the health benefits of tai chi for students in higher education. Prev Med Rep. 2016;3:103-12.

24. Oh J, Kim SJ, Kim S, Kang KA, Kan J, Bartlett R. Development and evaluation of flipped learning using film clips within a nursing informatics course. Jpn J Nurs Sci. 2019;16(4):385-95. 
25. Kim HS, Kim MY, Cho MK, Jang SJ. Effectiveness of applying flipped learning to clinical nursing practicums for nursing students in Korea: A randomized controlled trial. Int J Nurs Pract. 2017;23:5.

26. Ya-Qian L, Yu-Feng L, Meng-Jie L, Peng-Xi L, Julie T, Li-Na M, et al. Effectiveness of the flipped classroom on the development of self-directed learning in nursing education: a meta-analysis. Front Nurs. 2018;5(4):317-29.

27. Im S, Jang SJ. Effects of a clinical practicum using flipped Learning among undergraduate nursing students. J Nurs Educ. 2019;58(6):354-6.

28. Kim YM, Yoon YS, Hong HC, Min A. Effects of a patient safety course using a flipped classroom approach among undergraduate nursing students: a quasi-experimental study. Nurse Educ Today. 2019;79:180-7.

29. Maxwell $\mathrm{KL}$, Wright $\mathrm{VH}$. Evaluating the effectiveness of two teaching strategies to improve nursing Students' knowledge, skills, and attitudes about quality improvement and patient safety. Nurs Educ Perspect. 2016; 37(5):291-2.

30. Holman R, Hanson AD. Flipped Classroom Versus Traditional Lecture: Comparing Teaching Models in Undergraduate Nursing Courses. Nurs Educ Perspect. 2016;37:6.

31. Kim H, Jang Y. Flipped Learning with simulation in undergraduate nursing education. J Nurs Educ. 2017;56(6):329-36.

32. Geist MJ, Larimore D, Rawiszer $H$, Sager AWA. Flipped versus traditional instruction and achievement in a baccalaureate nursing pharmacology course. Nurs Educ Perspect. 2015;36(2):114-5.

33. Dabney BW, Mitchell R. Flipping an undergraduate Gerontological nursing course: student perceptions. Nurs Educ Perspect. 2017;38(6):340-1.

34. El-Banna MM, Whitlow M, McNelis AM. Flipping around the classroom: accelerated bachelor of science in nursing students' satisfaction and achievement. Nurse Educ Today. 2017;56:41-6.

35. Simpson V, Richards E. Flipping the classroom to teach population health: increasing the relevance. Nurse Educ Pract. 2015;15(3):162-7.

36. Greenwood VA, Mosca C. Flipping the nursing classroom without flipping out the students. Nurs Educ Perspect. 2017;38(6):342-3.

37. Saunders A, Green R, Cross M. Making the most of person-centred education by integrating flipped and simulated teaching: an exploratory study. Nurse Educ Pract. 2017;27:71-7.

38. Green RD, Schlairet MC. Moving toward heutagogical learning: illuminating undergraduate nursing students' experiences in a flipped classroom. Nurse Educ Today. 2017:49:122-8

39. Bingen HM, Steindal SA, Krumsvik R, Tveit B. Nursing students studying physiology within a flipped classroom, self-regulation and off-campus activities. Nurse Educ Pract. 2019;35:55-62.

40. Cho M-K, Kim MY. Outcomes and influential factors applying flipped learning methods in a clinical adult nursing practicum. Int J Nurs Pract. 2019;25(2):e12724

41. Harrington SA, Bosch MV, Schoofs N, Beel-Bates C, Anderson K. Quantitative Outcomes for Nursing Students in a Flipped Classroom. Nurs Educ Perspect. 2015;36:3.

42. Park EO, Park JH. Quasi-experimental study on the effectiveness of a flipped classroom for teaching adult health nursing. Jpn J Nurs Sci. 2018;15(2):125-34.

43. Hanson J. Surveying the experiences and perceptions of undergraduate nursing students of a flipped classroom approach to increase understanding of drug science and its application to clinical practice. Nurse Educ Pract. 2016;16(1):79-85.

44. Xu P, Chen Y, Nie W, Wang Y, Song T, Li H, et al. The effectiveness of a flipped classroom on the development of Chinese nursing students' skill competence: a systematic review and meta-analysis. Nurse Educ Today. 2019;80:67-77.

45. Lee J, Lim C, Kim H. Development of an instructional design model for flipped learning in higher education. Educ Technol Res Dev. 2017;65(2): 427-53.

46. Smith CM, McDonald K. The flipped classroom for professional development: part II. Making podcasts and videos. J Contin Educ Nurs. 2013 44(11):486-7.

47. Strayer JF. How learning in an inverted classroom influences cooperation, innovation and task orientation. Learn Environ Res. 2012;15(2):171-93.

48. Garrison DR, Kanuka H. Blended learning: uncovering its transformative potential in higher education. Internet High Educ. 2004;7(2):95-105.

49. Nuray G, Ercan K, Ozden MY. Design of a blended learning environment Considerations and implementation issues. Aust J Educ Technol. 2013;29:1.

50. Herreid CF, Schiller NA. Case studies and the flipped classroom. J Coll Sci Teach. 2013;42(5):62-6.
51. Arnold I. John Hattie: visible learning: a synthesis of over 800 meta-analyses relating to achievement. Int Rev Educ. 2011;57(1):219.

52. Roehl A, Reddy SL, Shannon GJ. The flipped classroom: an opportunity to engage millennial students through active Learning. J Fam Consum Sci. 2013;105(2):44-9.

53. Talbert R. Learning MATLAB in the Inverted Classroom. 2012 ASEE Annual Conference \& Exposition; San Antonio, Texas. San Antonio ASEE Conferences; 2012. p. 25.883.1-25..19.

54. Hsu TC, Lee-Hsieh J, Turton MA, Cheng SF. Using the ADDIE model to develop online continuing education courses on caring for nurses in Taiwan. J Contin Educ Nurs. 2014:45(3):124-31.

55. Lu SC, Cheng YC, Chan PT. Using ADDIE model to develop a nursing information system training program for new graduate nurse. Stud Health Technol Inform. 2016;225:969-70.

56. Cheung L. Using the ADDIE model of instructional design to teach chest radiograph interpretation. J Biomed Educ. 2016:9502572.

57. Reinbold S. Using the ADDIE model in designing library instruction. Med Ref Serv Quart. 2013;32(3):244-56.

58. Kim MK, Kim SM, Khera O, Getman J. The experience of three flipped classrooms in an urban university: an exploration of design principles. Internet High Educ. 2014;22:37-50.

59. Bakr MM, Massey WL, Massa HM. Flipping a dental anatomy course: a retrospective study over four years. Educ Res Int. 2016:7097398.

60. Galway LP, Corbett KK, Takaro TK, Tairyan K, Frank E. A novel integration of online and flipped classroom instructional models in public health higher education. BMC Med Educ. 2014;14(1):181.

61. Liebert CA, Lin DT, Mazer LM, Bereknyei S, Lau JN. Effectiveness of the Surgery Core Clerkship Flipped Classroom: a prospective cohort trial. Am J Surg. 2016;211(2):451-7 e1.

62. McLaughlin JE, Griffin LM, Esserman DA, Davidson CA, Glatt DM, Roth MT, et al. Pharmacy student engagement, performance, and perception in a flipped satellite classroom. Am J Pharm Educ. 2013;77(9):196.

63. Morton DA, Colbert-Getz JM. Measuring the impact of the flipped anatomy classroom: The importance of categorizing an assessment by Bloom's taxonomy. Anat Sci Educ. 2017;10(2):170-5.

\section{Publisher's Note}

Springer Nature remains neutral with regard to jurisdictional claims in published maps and institutional affiliations.
Ready to submit your research? Choose BMC and benefit from:

- fast, convenient online submission

- thorough peer review by experienced researchers in your field

- rapid publication on acceptance

- support for research data, including large and complex data types

- gold Open Access which fosters wider collaboration and increased citations

- maximum visibility for your research: over $100 \mathrm{M}$ website views per year

At BMC, research is always in progress.

Learn more biomedcentral.com/submissions 